\title{
Study On Distribution Characteristics Of Rated Power For Motor Truck
}

\author{
Guoliang Dong ${ }^{1, a^{*}}$, Fujia Liu ${ }^{2, b}$, ShuquanXu ${ }^{3, c}$ \\ Research Institute of Highway Ministry of Transport, Beijing, China \\ agl.dong@rioh.cn, bfj.liu@rioh.cn, csq.xu@rioh.cn
}

Keywords: motor truck, motor power, mismatch, hypothesis distribution, EDF, goodness test

\begin{abstract}
There are thousands of truck models in the market. It is necessary to study the distribution characteristics of motor power since the rated power mismatch is a very serious problem currently. The truck models of maximum total mas range from 5 tons to 7 tons have been analyzed. Construct statistics for different hypotheses distributions, estimate their values, test the goodness of these distribution hypotheses and determine their distribution characteristics.
\end{abstract}

\section{Introduction}

There are thousandsof truck models in the market.Improper configuration design will result in the power mismatch, weak safety, performance loss and service life decrease [1] etc. Currently, rated power mismatch is a very common and serious problem. In order to study present situation of power mismatch, we should acquire as many as possible truck models and sort out them by maximum total mass(MTM) to study distribution character of the motor power. We have analyzed the sample data of MTM range from 5 tons to 7 tons. Assuming that motor power follows some distributions, we construct statistics of various distributions, estimate values and test the goodness of these distribution hypotheses [2].

\section{Research Method}

Motor Truck Category. The proper motor power is relative to MTM. More MTM, more power. The MTM of motor truck vary from 3.5 tons to 31 tons. With constant sample size,a narrower MTM range will provide highprecision of the rated power average but reduce sample quantityfor each category. By reference to the category method of the fuel-consumption-limits standard[3] and other classification methods, motor truck models can be classified asalmost 2 tons interval for each categorywhich results in a proper precision with a necessary sample size for each category.

Sample. Truck parameters can be acquired from many ways such as books, truck plants, automotive websites [4], vehicle forums, auto 4S shops etc. The most authoritative data source is truck plant.As a good starti, we have acquired thousands of motor truck data of MTM range from 5 tons to 7 tons from truck plants. In order to eliminate distractionsof one model corresponding to various configurations (one model assembled with the same motor types), the same model with various configurations should be merged into one line.

Data Analysis.(1)Distribution character

By studying the scatter diagram and frequency chart of the rated power, the distributionof data focusing in the middle and decreasing on both sides is significant. According to the statistics theory,normal distribution,lognormal distribution, Weibull distribution and Gamma distribution comply with the above feature [5,6].

(2)Hypothesis distribution theory

There are many methods toconstructstatistic. The statistic based on the difference between the empirical distribution function (EDF) and the theoretical distribution function is called EDF statistic [7].For continuous vary, the test of goodness of fit is better than $\chi^{2}$ test. And EDF statistics have nothing to do with the group methods [8].Follow the three steps to study the distribution of motor power. (1) Compute the estimated parameter values of hypothesis function using maximum likelihood method (MLE). (2) Construct $A^{2}$-Statistics, $W^{2}$-Statistics and $D$-Statistics. (3) Test the 
goodness of fit check of the hypothesis distribution.

$D$-Statistics[9]:

$$
D_{n}=\sup _{\forall x \in R}\left[F_{n}(x)-F(x, \theta)\right]
$$

Where $\forall x$ denotes with any $x, R$ denotes the range over of $x, F_{n}(x, \theta)$ denotes the theoretical distribution function of $\mathrm{X}, \mathrm{F}_{\mathrm{n}}(\theta)$ denotes the empirical distribution function of $\mathrm{X}$.

$A^{2}$-Statistics, $W^{2}$-Statistics can be calculatedbyEq.2 and Eq.3[10,11].

$$
\begin{aligned}
& A^{2}=n \int_{-\infty}^{+\infty}\left[F_{n}(x)-F(x, \theta)\right]^{2}\{F(x, \theta)[1-F(x, \theta)]\}^{-1} d F(x, \theta) \\
& W^{2}=n \int_{-\infty}^{+\infty}\left[F_{n}(x)-F(x, \theta)\right]^{2} d F(x, \theta)
\end{aligned}
$$

Set $\mathrm{Z}=\mathrm{F}(\mathrm{X}, \theta), A^{2}$-Statistics, $W^{2}$-Statistics and, $D$-Statistics can be computedby Eq.4-Eq.6.

$$
\begin{aligned}
& \left\{\begin{array}{l}
D_{n}{ }^{+}=\max _{\forall i \in(1,2 \cdots n)}\left[\frac{i}{n}-z_{(i)}\right] \\
D_{n}{ }^{-}=\max _{\forall i \in(1,2 \cdots n)}\left[z_{(i)}-\frac{i-1}{n}\right] \\
D_{n}=\max \left[D_{n}{ }^{+}, D_{n}{ }^{-}\right]
\end{array}\right. \\
& \mathrm{W}_{\mathrm{n}}^{2}=\sum_{\mathrm{i}=1}^{\mathrm{n}}\left[\mathrm{z}_{(\mathrm{i})}-\frac{2 \mathrm{i}-1}{2 \mathrm{n}}\right]^{2}+\frac{1}{12 \mathrm{n}} \\
& \mathrm{A}_{\mathrm{n}}^{2}=-\frac{1}{\mathrm{n}}\left\{\sum_{\mathrm{i}=1}^{\mathrm{n}}(2 \mathrm{n}-1)\left[\ln \left[\mathrm{z}_{(\mathrm{i})}-\ln \left(1-\mathrm{z}_{(\mathrm{n}+1-\mathrm{i})}\right)\right]\right\}-\mathrm{n}\right.
\end{aligned}
$$

Considering that there are unknownvariables in parameters, EDF Statisticscanbe adjusted by the sample size as Table 1.

Table1.The adjusted EDF statisticsby sample size

\begin{tabular}{|l|c|c|c|}
\hline \multirow{2}{*}{ Normal } & \multicolumn{3}{|c|}{$T_{n}^{*}$, the correction of statistic $T_{n}$ by sample size } \\
\cline { 1 - 3 } & $A_{n}^{2}\left(1+\frac{0.75}{n}+\frac{2.25}{n^{2}}\right)$ & $W_{n}^{2}\left(1+\frac{0.5}{n}\right)$ & $D^{*}$ \\
\hline Lognormal & $A_{n}^{2}\left(1+\frac{0.2}{\sqrt{n}}\right)$ & $W_{n}^{2}\left(1+\frac{0.2}{\sqrt{n}}\right)$ & $D_{n}\left(\sqrt{n}-0.01+\frac{0.85}{\sqrt{n}}\right)$ \\
\hline Weibull & $A_{n}^{2}+\frac{1}{n}\left(0.2+\frac{0.3}{n}\right)$ & $\frac{1.8 n W_{n}^{2}-0.14}{1.8 n-1}$ & \\
\hline
\end{tabular}

Compare the statistic value with the adjusted value to judge if the EDF complying with the distribution hypotheses or not.

\section{Results And Discussion}

Data Processing.MATLABis an efficient tool for scientific computation.By editing some lines of programcode,we can compute parameter estimation of hypothesis distribution and EDF statistic. EDF statistic and the correction of rated power by sample size for $5 \mathrm{t}-7 \mathrm{t}$ motor truck are list in Table $2($ Sample size $=1209)$. 
Table 2EDF statistics and the correction of rated power by sample size for $5 \mathrm{t}-7 \mathrm{t}$ motor truck

\begin{tabular}{|c|c|c|c|c|c|c|}
\hline $\begin{array}{c}\text { statistic } \\
\text { hypothesis }\end{array}$ & $A^{2}$ & $W^{2}$ & $D$ & $A^{*}$ & $\mathrm{~W}^{*}$ & $D^{*}$ \\
\hline Normal & 6.0385 & 0.9502 & 0.0711 & 6.0422 & 0.9506 & 2.4732 \\
\hline Lognormal & 6.9044 & 1.2403 & 0.0933 & 6.9087 & 1.2408 & 3.2465 \\
\hline Weibull & 14.4958 & 2.2643 & 0.1070 & 14.5049 & 2.2652 & 3.7237 \\
\hline Gamma & 5.9760 & 1.0325 & 0.0848 & 5.9798 & 1.0330 & 2.9500 \\
\hline
\end{tabular}

Test results. The critical value at the significance level for the test statistics are list in Table 3 .

Table 3. The critical value at the significance level for the test statistics

\begin{tabular}{|c|c|c|c|}
\hline significance level $\alpha$ & $A^{2}$ & $W^{2}$ & $\sqrt{n D}$ \\
\hline 0.25 & - & 0.472 & 0.074 \\
\hline 0.10 & 0.819 & 0.632 & 0.102 \\
\hline 0.05 & 0.895 & 0.754 & 0.126 \\
\hline 0.025 & 0.995 & 0.875 & 0.148 \\
\hline 0.01 & 1.035 & 1.036 & 0.178 \\
\hline
\end{tabular}

Comparing the adjusted $A^{2} 、 W^{2}, D$ with the critical value for different significance level (If the statistics value is less than the critical value, the hypothesis distribution is accepted), we conclude that the distribution of rated power complies with no hypotheses distribution.

\section{Conclusion}

We have studied the distribution character and computed the EDF for each hypothesis distribution. But the results are not good.

By studying the distribution characteristics of sample models of $5 \mathrm{t}-7 \mathrm{t}$ motor truck, we can draw a conclusion as follows

The rated power doesn't conform to the assumed hypotheses distributionsof normal distribution and lognormal distribution.

The rated power doesn't follow a definite mathematical relationshipwith MTM

The motor plantshaven't followed the optimal design rules

\section{References}

[1] Zhisheng Yu,Theory of Automobile,China Machine Press,2009.

[2]Cong Dong,Haiwu Rong,Renwei Xia, The Distribution Model of Fatigue Life and its Good-of-Fit Test, Chinese J. of Aeronautics (1995),8(3),pp.185-190.

[3] JT 719-2008 Limits and Measurement Methods of Fuel Consumption for Commercial Vehicle for Cargos Transportation

[4]Information onhttp://www.xcar.com.cn/

[5] Zou Sheng, Shiqian Xie, Chengyi Pan,Probability and Mathematical Statistics, Higher Education Press,2010.

[6]Junping Jia,Statistics, China Renmin University Press Co.,LTD,2015.

[7]Zhenhai Yang,Weihu Cheng, Junjian Zhang,Goodness of Fit Test, Science Press,2001.

[8]Cong Dong, Modern structural system reliability theory and its application, Science Press,2001.

[9] Peter J.Bickel, Kjell A.Doksum, Mathematical StatisticsBasic Ideas and Selected Topics, Prentice-Hall,Inc.,2001. 
[10] Wei Zhang,Shenglin Yu,Gong Zhang, Anderson-Darling test for clutter distribution identification,Chinese Journal of Scientific Instrument, Vol.30,2009,pp.631-635

[11]Paul A. Tobias \& David C. Trindade,Applied Reliability,third ed.,CRC Press, Taylor \& Francis Group,2012. 